\title{
A study on trunk muscle activation patterns according to tilt angle during whole body tilts
}

\author{
Sun Hye Shin ${ }^{\mathrm{a}}$, Seung Rok Kang ${ }^{\mathrm{a}}$, Tae-Kyu Kwon ${ }^{\mathrm{b}, *}$ and Changho $\mathrm{Yu}^{\mathrm{c}, *}$ \\ ${ }^{a}$ Department of Healthcare Engineering, Chonbuk National University, Jeonju, Jeonbuk 54896, Korea \\ ${ }^{\mathrm{b}}$ Division of Biomedical Engineering, Chonbuk National University, Jeonju, Jeonbuk 54896, Korea \\ ${ }^{\mathrm{c}}$ Department of Convergence Technology Engineering, Chonbuk National University, Jeonju, Jeonbuk \\ 54896, Korea
}

\begin{abstract}
.
BACKGROUND: Lower back pain has a lifetime prevalence of $80 \%$ for people in a modern society, and in recent years, spinal stabilization movement has been highlighted as a method to treat lower back pain and an unbalanced position.

OBJECTIVE: The objectives of this study were to assess the trunk muscle activities in accordance with the tilt angles $\left(10^{\circ}\right.$, $20^{\circ}, 30^{\circ}, 40^{\circ}$ ) during trunk tilt exercises with a $3 \mathrm{D}$ dynamic exercise device.

METHODS: Active tilt mode (a self-generated active movement in the angle and direction steered by the user) was used in this study. The rotation capability enabled the investigation of the anterior (A), anterior right (AR), right (R), posterior right (PR), posterior (P), posterior left (PL), left (L), and anterior left (AL) tilt directions. EMG signals of the trunk (3 global muscles: rectus abdominis, RA, external obliques, EO, latissimus dorsi, LD, and 1 local muscle: multifidus, MF) muscles were obtained. RESULTS: The MF muscle activity was higher while the anterior tilt was maintained, and the abdominal muscle activity was higher while the posterior tilt was maintained. Also, as the tilt angle increased, the activity of the muscles opposite the tilt direction increased.

CONCLUSIONS: The results indicate that to maintain equilibrium through posture stabilization during whole body tilting, the human body maintains a proper interaction among the body segments as well as between the body and the execution environment. Moreover, stability is maintained through the co-contraction of antagonistic and agonistic muscles. In future studies, it will be important to conduct research on improving imbalance in the trunk muscles.
\end{abstract}

Keywords: Trunk stabilization exercise, whole body tilt, tilt angle, EMG

\section{Introduction}

Postural imbalance is caused by repetitive tasks in modern people because they have to sit down and work for long hours, and they experience various occupational musculoskeletal injuries and backache due to the excessive use of local joints [1,2]. Recently, spinal stabilization exercises have been emphasized as a treatment for backache and postural imbalance, and various researches on spine stabilization has been carried out. Spine stabilization refers to the ability to control major or minute movements of

\footnotetext{
${ }^{*}$ Corresponding author: Tae-Kyu Kwon, Division of Biomedical Engineering, Chonbuk National University, 567 Baekjedaero, Deokjin-gu, Jeonju-si, Jeonbuk 54896, Korea. Tel.: +82 632704066; Fax: +82 632702247; E-mail: kwon10@jbnu.ac.kr and Changho Yu, Department of Convergence Technology Engineering, Chonbuk National University, 567 Baekje-daero, Deokjin-gu, Jeonju-si, Jeonbuk 54896, Korea. Tel.: +82 634722898; Fax: +82 632704226; E-mail: combo418@ nate.com.
}

0928-7329/17/\$35.00 (c) 2017 - IOS Press and the authors. All rights reserved

This article is published online with Open Access and distributed under the terms of the Creative Commons Attribution NonCommercial License (CC-BY-NC 4.0). 
spinal joints consciously or unconsciously, and stabilization exercise strengthens the simultaneous contraction abilities of muscles and spinal muscular strength to maintain stability in a spine-neutral posture, adapting well to the load applied to the spine, that it is designed to recover muscle strength and movement control capabilities [3-7].

The trunk muscles are classified into local and global muscles. Local muscles, such as the multifidus and transversus abdominis, are the innermost muscles, that maintain a neutral posture and stabilize the spinal segment during activities, providing dynamic stability during co-contraction, and global muscles, located near the surface, such as the internal oblique abdominis, external oblique abdominis, and lumbar quadrate muscles, surrounding the lumbar spine, generate stabilization and movement [8-10]. While the roles of local muscle attached closest to the spine were emphasized or only the roles of global muscles were emphasized in terms of exercise in the past, recent research results have identified the importance of stabilization exercises through co-contraction of local and global muscles [9,11-13].

Also, the exercises as described by Williams and McKenzie, widely known as spine stabilization exercises [10,22-24], are simple contraction/expansion exercise; however, they have been assessed as inappropriate for stability enhancement because they generate excessive loads, with repetitive stress, tiredness, and microtrauma. More precise and quantitative spine stabilization devices that allow exercises in various patterns, considering the human body three-dimensionally have been developed recently [14, 15].

Anders et al. analyzed muscle activity in the leaning motion of the human body using Centaur, and measured and compared the muscle activation according to gender and age during the leaning motion in horizontal and vertical directions [16,17]. Also, Kim et al. suggested effective exercise methods of spine stabilization by evaluating the muscle activity depending on the axis rotation during front and back leaning with three-dimensional (3D) lumbar stabilization devices [11]. Kang et al. analyzed the effects of visual biofeedback during whole-body leaning exercises for maintaining correct posture and muscular activity of trunk muscles [18]. Yu et al. analyzed the trunk and leg muscle activities during the trunk tilt exercise by a 3D dynamic exercise device to investigate the influence of the trunk positions, but the tilt angle was fixed at $30^{\circ}$. Moreover, they didn't investigate of the muscular pattern according to the different tilt angle [19].

Thus, this study was intended to compare and analyze changes in the trunk muscle activity using 3D dynamic exercise equipment while maintaining the leaning of the human body in eight directions and four angles by helping users to maintain direction and angle without using any device.

\section{Methods}

\subsection{Subjects}

For this study, 10 healthy volunteers ( 10 males, height $175 \pm 2.86 \mathrm{~cm}$, mass $68.3 \pm 3.2 \mathrm{~kg}$, age $24.5 \pm$ 0.6 years) participated. All subjects were healthy and had no orthopedic problems of the spine or legs muscles. All subjects, consistent with the Declaration of Helsinki (1964), volunteered after signing a written informed consent. The investigation was performed with a device for trunk muscle diagnosis and training (Spine Balance 3D, CyberMedic Co. and Chonbuk National University, Korea; Fig. 1).

\subsection{System configuration}

Figure 1(a) shows a 3D dynamic exercise device (Spine Balance 3D) capable of moving $100^{\circ}$ in the 

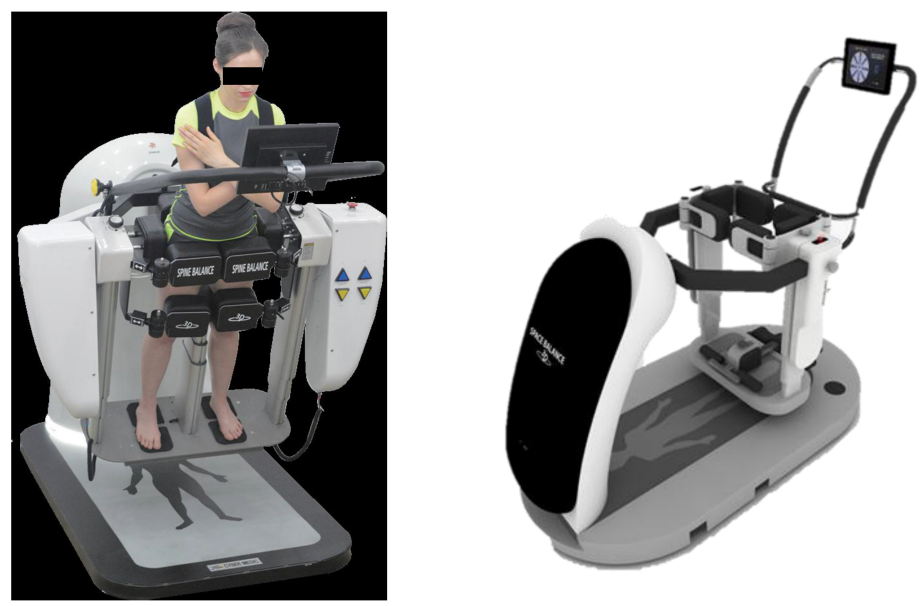

(a)

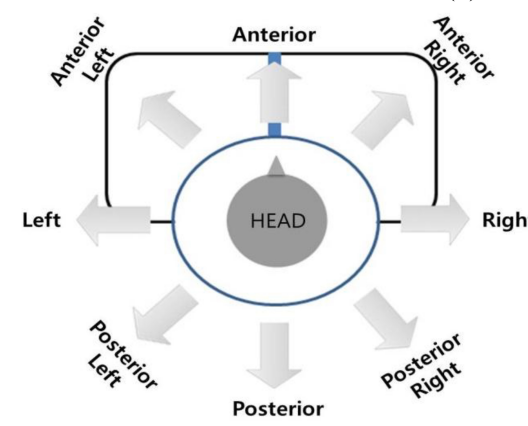

(b)

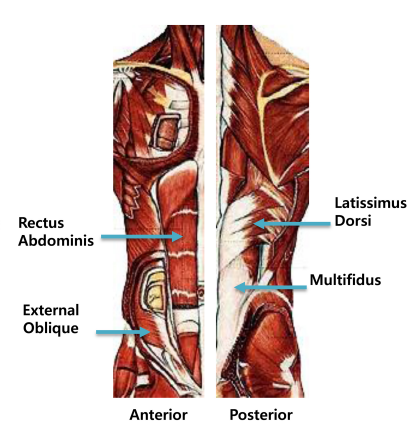

(c)

Fig. 1. (a) 3D dynamic exercise device we developed (Spine Balance 3D, CyberMedic Co. and Chonbuk National University, Korea), (b) Explanation of the eight tilting directions during the trunk tilt exercise modes with the device, (c) Trunk muscles measure to verify muscular activity.

anterior-posterior direction, and $180^{\circ}$ in the left-right direction. Thus, it can be rotated in the anteriorposterior and left-right and diagonal direction and in 3D space. Also, the device has two exercise modes: a self-generated active movement in the angle and direction steered by the user (active tilt mode), and a passive movement generated by the driving of the equipment (passive tilt mode) [19]. In this study, we used only the active tilt mode.

The device included various exercise program modes for exercise and diagnosis of balance and trunk issues. As shown in Fig. 1(b), with the diagnosis program mode for the evaluation of whole body tilt ability, we measured the trunk muscle generated by tilting in eight different directions - A (anterior), R (right), P (posterior), L (left), AR (anterior right), AL (anterior left), PR (posterior right), and PL (posterior left) - during the diagnosis modes of the device. This device applies forces and balances on the trunk by tilting the whole body from a neutral upright position. The subject is fixed at the feet and pelvis, but the trunk remains unsupported. During tilt, the subject simply has to stabilize his upper body in the body axis. Also, subjects held their arms crossed against their chests. Exact body and arm positioning throughout the whole investigation was controlled by the examiner.

For the record and analysis of the EMG signals, we was used an 8-channel surface EMG system (Bignoli system, Delsys. Inc., USA). All raw EMG signals were band pass-filtered between 6 and $400 \mathrm{~Hz}$ and then amplified. The sampling rate was $1000 \mathrm{~Hz}$ and the signals were converted from an analog 
voltage to a digital signal at $1000 \mathrm{~Hz}$ (A/D conversion) before being entered into a personal computer. The analysis method used root-mean-square (RMS) values. RMS values were calculated to quantify the amplitude of the EMG signals. Electrodes were attached after removing the keratinous skin layer with sterilization alcohol to reduce skin resistance against the surface EMG signals. We measured four trunk muscles during the exercise. Four muscles ( 3 global muscles and 1 local muscle) were assessed simultaneously from both sides of the body: rectus abdominis, external obliques, latissimus dorsi, and multifidus (Fig. 1(c)).

\subsection{Experiment and analysis}

Our experimental procedures were conducted by explaining the analysis of trunk and activities generated by four angles in eight tilting directions, in a self-generated active movement with the angle and direction steered by the user. The tilting directions were anterior $(\mathrm{A})$, right $(\mathrm{R})$, posterior $(\mathrm{P})$, left $(\mathrm{L})$, anterior right (AR), anterior left (AL), posterior right (PR), and posterior left (PL), and angles are $10^{\circ}$, $20^{\circ}, 30^{\circ}$, and $40^{\circ}$. The tilting directions were applied randomly; the subjects simply had to stabilize his upper body in the body axis. Subjects maintained their body with the eight tilting directions. Each session consisted of two repetitions of $10 \mathrm{~s}$ duration to ensure stationary conditions for at least $30 \mathrm{~s}$. To recover muscular strength, 20 min of rest was allotted between directions and angles.

We measured trunk ( 3 global muscles: rectus abdominis, external obliques, latissimus dorsi, and 1 local muscle: multifidus) muscles during the exercises. Data were analyzed using the PASW statistics software (ver. 12 for Windows; SPSS Inc., Chicago, IL, USA). The Kolmogorov-Smirnov test (K S test) was used to evaluate differences in EMG activity between angles for changes in the measured parameters. A $\mathrm{P}$ value of $<0.05$ was considered on indicate statistical significance.

\section{Results}

Figure 2 illustrates trunk muscle activities according to tilt angle generated by tilting in eight directions; the $y$-axes represent root mean square (RMS) values converted from the electromyography (EMG) data, and the $\mathrm{x}$-axes indicate the trunk muscles.

In the case of maintaining the anterior direction tilt (Fig. 2(a)), multifidus muscle activity was significantly higher according to increase of tilt angle, but rectus abdominis and external oblique muscle activities were significantly lower $(p<0.05)$. While maintaining the right direction tilt (Fig. 2(b)), left rectus abdominis, external oblique, and multifidus muscle activities were significantly higher according to increase of tilt angle, whereas right muscle activity was not significantly different.

According to the RMS values of maintaining of the posterior direction tilt (Fig. 2(c)), rectus abdominis and external oblique muscle activities were significantly higher according to the increase in tilt angle. However, multifidus muscle activity was not significantly different, despite an increase in tilt angle. According to the RMS values for maintaining a left direction tilt (Fig. 2(d)), the right rectus abdominis, external oblique, and multifidus muscle activity was significantly higher according to the increase in tilt angle, whereas left muscle activity was not significantly different. In the case of maintaining the anterior right direction tilt (Fig. 2(e)), the left external oblique of the abdominal muscles and the left multifidus of the back muscle activity showed co-contraction; multifidus muscle activity was significantly higher.

In the case of maintaining a posterior right direction tilt (Fig. 2(f)), the rectus abdominis and external oblique abdominal muscle activities were significantly higher according to the increase in tilt angle; especially the left abdominal muscles activity was significantly higher than the right abdominal muscles. 

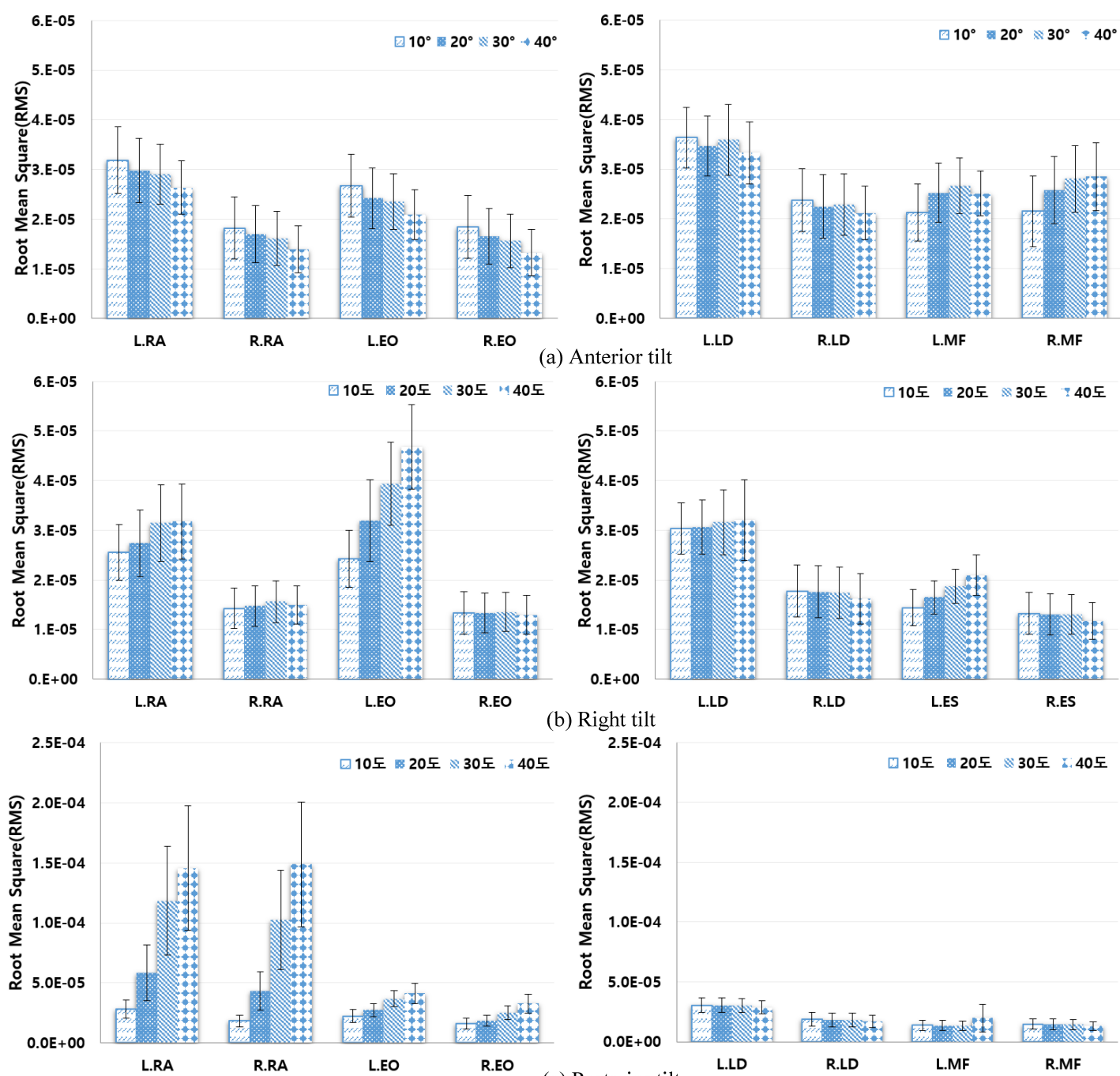

(b) Right tilt
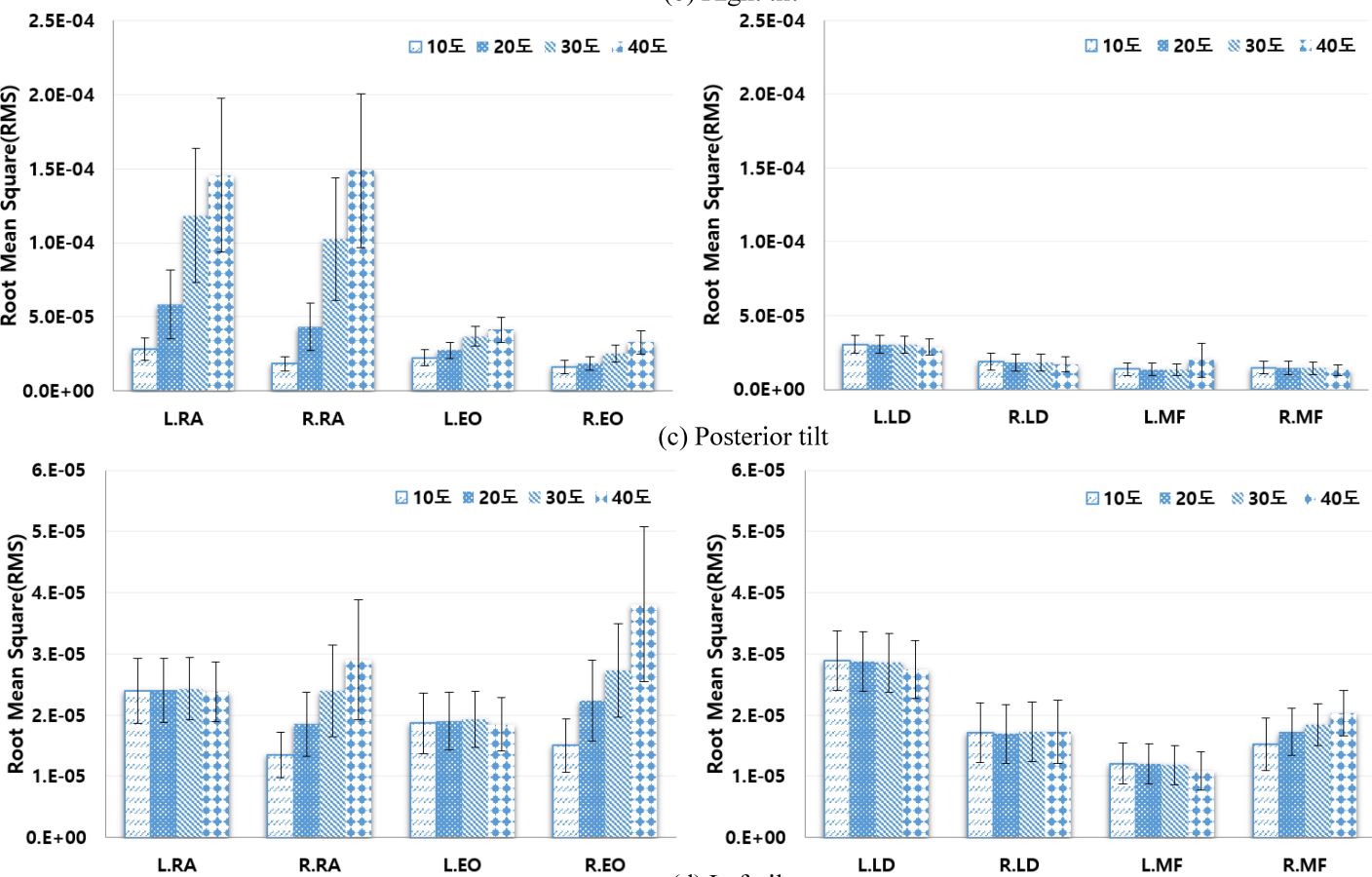

(c) Posterior tilt

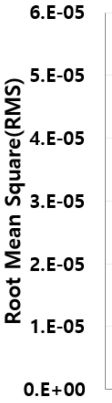

(d) Left tilt

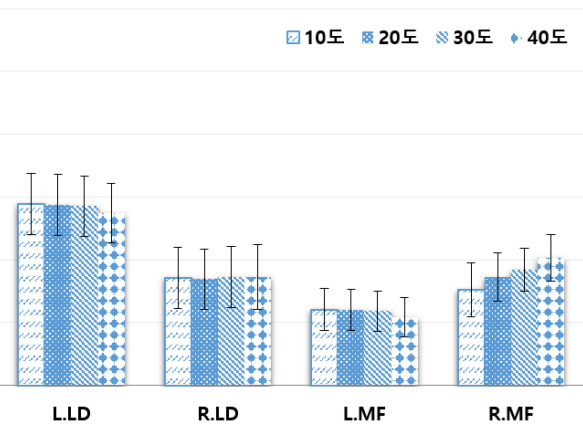

Fig. 2. Results of trunk muscle activities of eight trunk muscles according to four tilt angles during whole-body tilt. Abbreviations: left rectus abdominis $=$ L.RA, right rectus abdominis $=$ R.RA, left external oblique $=$ L.EO, right external oblique $=$ R.EO, left latissimus dorsi $=$ L.LD, right latissimus dorsi $=$ R.LD, left multifidus $=$ L.MF, right multifidus $=$ R.MF. 

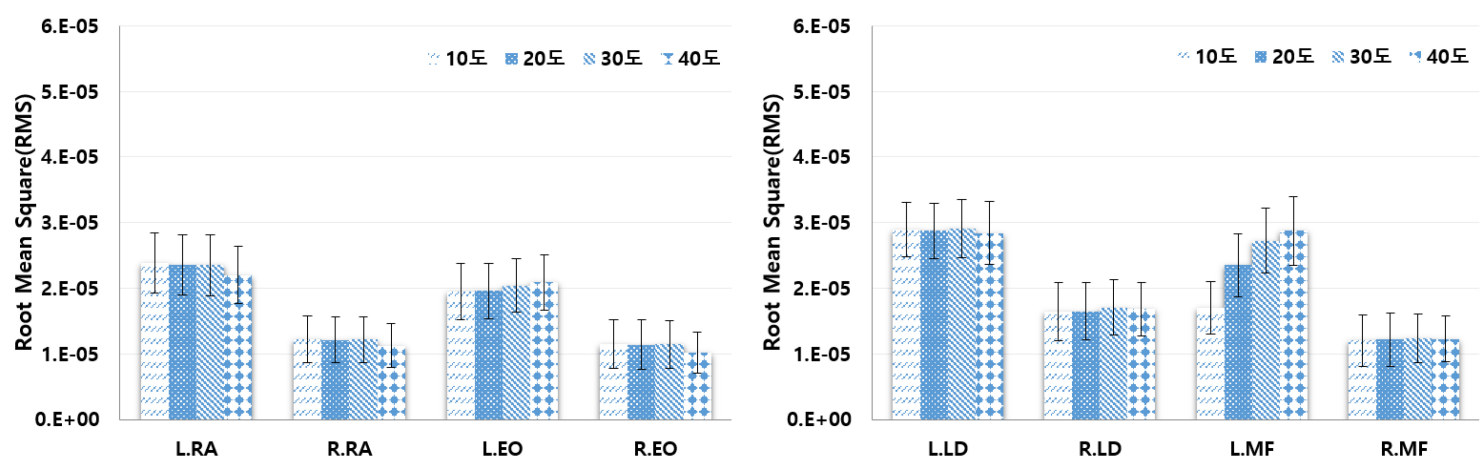

(e) Anterior Right tilt

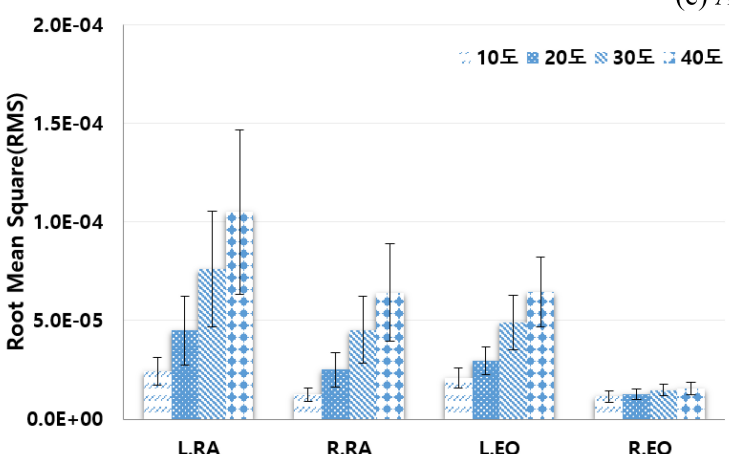

$$
\text { 2.0E-04 }
$$
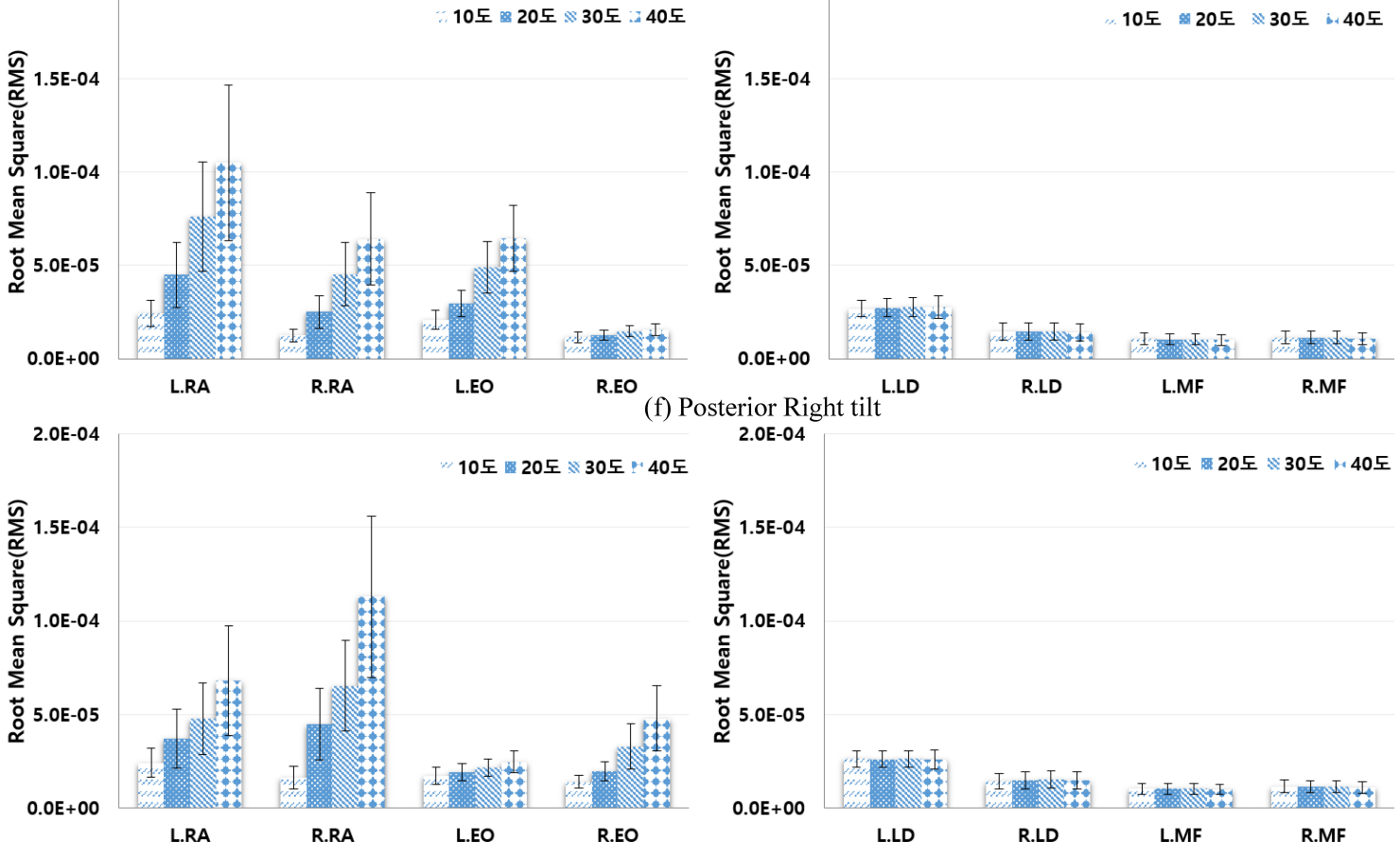

10도 原 20도 $\ll 30$ 도 $\cdots 40$ 도

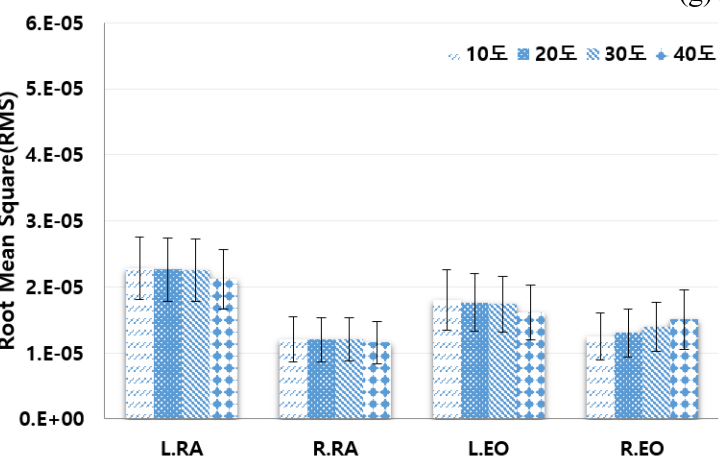

(g) Posterior Left tilt

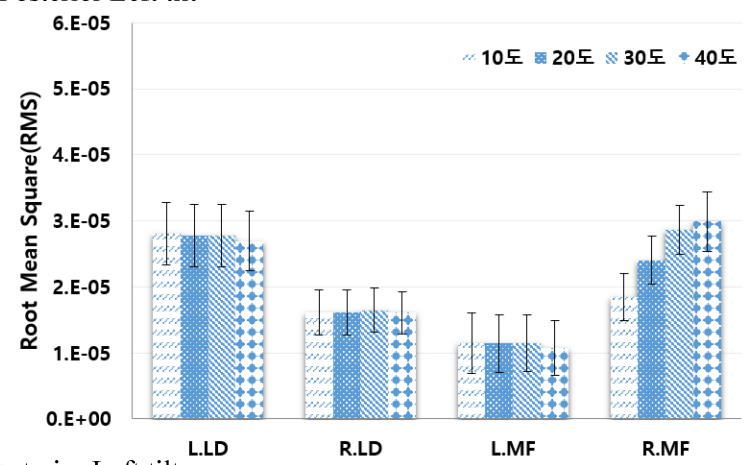

(h) Anterior Left tilt

Fig. 2. continued. 
While maintaining a posterior left direction tilt (Fig. 2(g)), similar to the posterior right tilt, the rectus abdominis and external oblique abdominal muscle activities were significantly higher according to the increase in tilt angle. However, right abdominal muscle activity was significantly higher than the left abdominal muscles.

While maintaining an anterior left direction tilt (Fig. 2(h)), the right external oblique abdominal muscles and right multifidus back muscle activities showed co-contraction, and especially right multifidus muscle activity was significantly higher. However, in terms of the left muscles, there was no significant difference in the tilt angle. Additionally, no significant difference was seen in latissimus dorsi muscle activity according to tilt direction or angle.

\section{Discussion}

This study shows basic results for comparing differences in trunk muscle activity depending on eight types of inclined direction and four angles and contraction activity of the trunk muscles was analyzed comparatively using a 3D dynamic exercise outfit in which inclination retention is allowed by voluntary movements of the test subject.

When observing the results of trunk muscle activity, depending on inclined angle, as the angle increased, muscle activity on the opposite side to the incline direction increased significantly but in all the muscles on the inclined direction and movement side, excluding the abdominal muscles, muscle activity change was not represented at the time of forward inclination. This phenomenon is considered to indicate that the more the inclined angle increases, the more the activity of the muscles on the opposite side also increase, because those muscles contract so that human body may maintain its inclination without losing balance while muscles on the opposite side of inclined direction becomes agonistic muscles in maintaining the inclination.

Additionally, it is considered that the human body simultaneously contracts antagonistic muscles that generates moments in opposite directions to overcome outside moments and muscles in the same direction as that of inclination are antagonistic muscle and contract simultaneously with agonistic muscle to increase stability and effectively respond to changes in posture and internal and external moments [20]. That is, it is believed that while human body inclination is maintained at the time of maintaining inclination to in a specific direction, agonistic muscles are activated together with antagonistic muscles. In particular, in the case of the rectus abdominis and external oblique, the muscle activity showed a tendency to decrease as the angle was increased in the forward direction, contrary to the other direction. This indicates that as test subject used his muscles after moving the center of gravity at the time of forward inclination, if the inclined angle is large, the center of gravity movement of the human body is increased and so, muscle activity of rectus abdominis is relatively decreased.

Whereas we have seen some high activated muscles depending on tilting angle and direction, partial muscles help keep the body balance while maintaining the low muscle activation. For example, while maintaining the back direction tilt, not only abdominal muscles have high activity but also multifidus muscle has low activity. The finding considered that human body tries to maintain the tilt or the postural balance through muscles co-contraction of local and global muscles. Benedicte et al. investigated the influence of velocity on both the relative muscle activity and the ratio local to global muscle activity of the back and abdominal muscles. This results in a significant higher multifidus to iliocostalis pars thoracis ratio at higher velocities [25]. However, this study cannot explain the ratio local to global muscle activity during whole body tilt, because we just focused on the different muscle activation patterns. 
When observing muscle activity depending on direction, muscle activity of the multifidus was found to be high at the time of maintaining forward inclination. Conversely, at the time of maintaining rear inclination, the abdominal muscles were gradually, increasingly activated and it could be realized that at the time of left inclination, the right muscle was used and, at the time of right inclination, the left muscle was used [11,16,17,21]. Results of muscle activities, depending on each direction, were similar to that of this, indicating that to maintain equilibrium through posture stabilization, the human body maintains a proper interaction among body segments and between the human body and external environment [21].

In the case of the latissimus dorsi, directional difference was not seen because in movement to maintain inclination, activity of latissimus dorsi was restricted by requiring movement with the arms in a crossed condition in front of the chest.

The results have shown that exercise may be possibility of training for adolescent with scoliosis patient. Pugacheva assessed effectiveness of special corrective exercise in multimodality conservative therapy of idiopathic scoliosis, apply three dimensional trunk correction with side-shifting postural rotational were specified in accordance with type of scoliotic deformation [26]. Also, Lewis suggested effective exercise methods of a patient with scoliosis using ATM2 ${ }^{\mathrm{TM}}$ devices and showed significantly improved treatment by reversing spinal curvature with the ATM2 ${ }^{\mathrm{TM}}$ [27]. Therefore, further study need prove to effects at improvement with scoliosis of trunk tilting exercise using 3D dynamic exercises device.

\section{Conclusion}

In this study, it could be appreciated that by comparatively analyzing trunk muscle activity during the time of maintaining human body inclination, based on diversified angles and directions, an interaction took place between the body location and the movement execution environment to maintain equilibrium and the human body maintains stability through simultaneous contraction of antagonistic and agonistic muscles. This study provides basic data for 3D dynamic exercises and comparatively analyzed trunk muscle activity, depending on direction and angle. In future studies, muscle imbalance and posture balancing ability could be improved at the time of applying an actual inclined movement.

\section{Acknowledgments}

This research was supported by Basic Science Research Program through the National Research Foundation of Korea (NRF) funded by the Ministry of Education (2015R1D1A1A01059525), by research funds for newly appointed professors of Chonbuk National University in 2016 and by National Research Foundation of Korea (NRF) grant funded by the Korea government (MSIP) (2016M3C1B6929838).

\section{Conflict of interest}

None to report.

\section{References}

[1] Wang XD, Wang HJ. Establishment of individual exercise therapy model for low back pain. 2009 2nd International Symposium on Knowledge Acquisition and Modeling (KAM). Wuhan. 2009; 122-125. 
[2] Anthony HW. Diagnosis and management of low back pain and sciatica. AM Fam Physician. 1995; 52: 1333-1341.

[3] Imai A, Kaneoka K, Okubo Y, Shiina I, Tatumura M, Izumi S, Shiraki H. Trunk muscle activity during lumbar stabilization exercises on both a stable and unstable surface. J Orthop Sports Phys Ther. 2010; 40: 369-375.

[4] Yang SR. The comparison of exercise method on back pain disability index, range of motion and muscle strength in low back pain patients. Master's thesis. Ulsan University: Ulsan; 2009.

[5] Kim LC. The effects of core stability exercise with physioball and floor exercise on electromyographic activity of selected trunk and abdominal muscles and balance in healthy adults. Master's degree. Dankook University: Cheonan; 2004.

[6] Han KS, Yu CH, Kwon TK. Muscle forces and activation patterns in the spine during spine stabilization exercise using a whole body tilt device. IJPEM. 2014; 15: 2589-2595.

[7] Yu CH, Piao YJ, Kim K, Kwon, TK. The effects of an 8-weeks training on postural control for the elderly. IJPEM. 2014; 15: 161-168.

[8] Moseley GL, Hodges PW, Gandevia SC. Deep and superficial fibers of the lumbar multifidus muscle are differentially active during voluntary arm movements. Spine. 2002; 27: 29-36.

[9] Cholewicki J and McGill SM. Mechanical stability of the in vivo lumbar spine: implications for injury and chronic low back pain. Clinical Biomechanics. 1996; 11: 1-15.

[10] Vollowitz E. Furniture prescription for the conservative management of low-back pain. Topic in Acute Care and Trauma Rehabilitation. 1988; 2: 18-37.

[11] Kim SB, Chang YH, Kim SK, Bae TS, Mun MS, Park JC. Changes of muscle activation pattern of trunk muscles during whole-body tilts with and without axial rotation. J Korean Soc Precis Eng. 2012; 29: 805-810.

[12] Bergmark A. Stability of the lumbar spine: a study in mechanical engineering. Acta Orthopaedica. 1989; 60: 1-54.

[13] Panjabi M, Abumi K, Duranceau J, Oxland T. Spinal stability and intersegmental muscle forces. a biomechanical model. Spine. 1989; 14: 194-200.

[14] Nam TK. The biomechanical analysis for the effects of supine to standing by spiral direct exercise for trunk stabilization, department of physical education. Doctor's degree. Chungnam National University: Chungnam; 2007.

[15] Akuthota V, Nadler SF. Core strengthening. Arch Phys Med Rehabil. 2004; 85: 86-92.

[16] Anders C, Brose G, Hofmann GO, Scholle HC. Gender specific activation patterns of trunk muscles during whole body tilt. Eur J Appl Physiol. 2007; 101: 195-205.

[17] Anders C, Brose G, Hofmann GO, Scholle HC. Evaluation of the EMG-force relationship of trunk muscles during whole body tilt. J Biomech. 2008; 41: 333-339.

[18] Kang MH, Yoon JY, Yang JL, Jung DH, Oh JS. The effect of visual biofeedback on EMG activity of trunk muscles and endurance holding time for correct position during whole-body tilt exercise. PTK. 2011; 18: 9-17.

[19] Yu CH, Shin SH, Jeong HC, Go DY, Kwon TK. Activity analysis of trunk and leg muscles during whole body tilt exercise. Biomed Mater Eng. 2014; 24: 245-254.

[20] Song YW, Jeong MG. Analysis of functional roles of ten trunk muscles in voluntary isometric exertion tasks. JESK. 2003; 22: 47-57.

[21] Shin SH, Yu M, Jeong GY, Yu CH, Kim K, Jeong HC, Kwon TK. The assessment on electromyography of trunk muscle according to passive and active trunk tilt exercise of 3-D dynamic postural balance training system. J Korean Soc Precis Eng. 2013; 30: 331-339.

[22] Esther JO. Therapeutic exercise in the management of non-specific low back pain. Low Back Pain, Dr. Ali Asghar Norasteh (Ed.). ISBN: 978-953-51-0599-2. 2012; 225-246.

[23] Battie MC, Cherkin DC, Dunn R, Clol MA, Wheeler KJ. Managing low back pain: attitudes and treatment preferences of physical therapists. Physical Therapy. 1994; 74219-226.

[24] Y1'lmaz F, Y''lmaz A, Merdol F, Parlar D, Sahin F, Kuran B. Efficacy of dynamic lumbar stabilization exercise in lumbar microdiscectomy. J Rehabil Med. 2003; 35: 163-167.

[25] Van Damme BB, Stevens VK, Van Tiggelen DE, Duvigneaud NN, Neyens E, Danneels LA. Velocity of isokinetic trunk exercises influences back muscle recruitment patterns in healthy subjects. Journal of Electromyography and Kinesiology. 2012; 23: 378-386.

[26] Pugacheva N. Corrective exercises in multimodality therapy of idiopathic scoliosis in children - analysis of six weeks efficiency-pilot study. Stud Health Technol Inform. 2012; 176: 365-371.

[27] Lewis C. A Review of Non-Invasive Treatment Interventions for Spinal Deformities. Physical Therapy Perspectives in the 21st; Century-Challenges and Possibilities. ISBN 978-953-51-0459-9. 2012; 67-88. 